ApJ, to appear

\title{
Shear Acceleration in Expanding Flows
}

\author{
F.M. Rieger \\ ZAH, Institut für Theoretische Astrophysik, Universität Heidelberg, Philosophenweg 12, 69120 \\ Heidelberg, and \\ Max-Planck-Institut für Kernphysik, P.O. Box 103980, 69029 Heidelberg, Germany \\ frank.rieger@mpi-hd.mpg.de \\ and \\ P. Duffy \\ University College Dublin, Belfield, Dublin 4, Ireland \\ peter.duffy@ucd.ie
}

\begin{abstract}
Shear flows are naturally expected to occur in astrophysical environments and potential sites of continuous non-thermal Fermi-type particle acceleration. Here we investigate the efficiency of expanding relativistic outflows to facilitate the acceleration of energetic charged particles to higher energies. To this end, the gradual shear acceleration coefficient is derived based on an analytical treatment. The results are applied to the context of the relativistic jets of active galactic nuclei. The inferred acceleration timescale is investigated for a variety of conical flow profiles (i.e., power law, Gaussian, Fermi-Dirac) and compared to the relevant radiative and non-radiative loss timescales. The results exemplify that relativistic shear flows are capable of boosting cosmic-rays to extreme energies. Efficient electron acceleration, on the other hand, requires weak magnetic fields and may thus be accompanied by a delayed onset of particle energization and affect the overall jet appearance (e.g., core, ridge line and limb-brightening).
\end{abstract}

Subject headings: Outflow, jets: general - Particle acceleration: shear - AGN

\section{Introduction}

The non-thermal radiation seen from astrophysical objects bears witness to the presence of energetic charged particles that have experienced efficient acceleration within these sources. In the 
galactic domain, new high-resolution observations of supernova remnants have brought fresh momentum to the theory of diffusive shock acceleration (e.g., Bell 2013, for review), while short-term variability seen in the context of active galactic nuclei (AGN) has motivated deeper studies of oneshot (gap- or reconnection-type) particle acceleration scenarios (see e.g., Rieger \& Aharonian 2012 , for review of the case of M87). Complementary, new observational results in the radio and VHE domain and progress in our understanding of turbulence modeling have given new impetus to turbulent shear acceleration and emission scenarios (e.g., Aloy \& Mimica 2008; Sahayanathan 2009; Liang et al. 2013, Grismayer et al. 2013; Ohira 2013; Laing \& Bridle 2013). The present study focuses on the potential of accelerating energetic charged particles in expanding relativistic outflows in a regime appropriate for Active Galactic Nuclei (AGN). It follows an earlier analysis where the implications for the high-speed (bulk flow Lorentz factors $\gamma_{b} \gtrsim 100$ ) gamma-ray-burst (GRB) regime has been investigated (Rieger \& Duffy 2005), provides a general derivation of the relations presented there, and extends it to the AGN context.

In contrast to GRBs, AGN are in fact seen to exhibit relativistic outflows extending up to hundreds of kilo-parsecs. In particular, for the "blazar" sub-class of AGN, radio VLBI/VLBA observations of their inner (pc-scale) jets frequently reveal significant apparent superluminal motion $\left(v_{a}>c\right)$ of individual jet components propagating away from the core. When the fastest measured radio jet components are put together, the velocity distribution shows a peak $\beta_{a}=v_{a} / c \sim 10$, with a tail extending up to $\sim 50$ (Lister et al. 2009, Piner et al. 2012), suggesting that (radio) flow speeds in AGN jets may reach bulk flow Lorentz factors up to $\gamma_{b} \sim \beta_{a} / \beta \sim 50$. On the other hand, measured speeds of the (parsec-scale) radio components in the VHE-detected, highfrequency-peaked BL Lac objects (HBLs) appear to be consistently well below those found in the above-noted, radio-selected samples (Piner \& Edwards 2013). If representative, the apparent lack of significant superluminal speeds in the parsec-scale radio jets of TeV-HBLs in fact would seem to suggest that the (radio) bulk flow Lorentz factor in these objects is only modest $\left(\gamma_{b} \sim 2-3\right)$, in contrast to common constraints on their (sub-pc-scale) bulk Lorentz (Doppler) factors based on radiative modeling of their nuclear high-energy emission. In principle, such a difference could be accounted for by some change in directionality (e.g., jet bending, intrinsic helical motion) or by the presence of some velocity gradient in the flow, such as a (longitudinally) decelerating flow (Georganopoulos \& Kazanas 2003, Levinson 2007), or a (transversally) structured jet with a fast-moving spine and a slower moving sheath (Ghisellini et al. 2005). The former might be caused by radiative Compton drag, while the latter scenario of a fast inner flow (spine) encompassed by a slower outer flow (sheath) is generically expected in MHD models for the formation of relativistic jets with an ergospheric-driven jet surrounded by a slow moving disk wind (see e.g. McKinney 2006; Porth \& Fendt 2010, Hawley et al. 2015). The implied flow velocity gradients could possibly facilitate the acceleration of energetic charged particles to higher energies once, e.g., particles are efficiently scattered across the flow (Rieger \& Duffy 2006), and this is what is studied here. 


\section{Shear acceleration in spherical coordinates}

In the comoving frame $K^{\prime}$, the acceleration coefficient in a gradual shear flow can be cast into the form (e.g., Webb 1989, eq. 3.27)

$$
\left\langle\frac{\Delta p^{\prime}}{\Delta t^{\prime}}\right\rangle_{s h}=\frac{1}{p^{\prime 2}} \frac{\partial}{\partial p^{\prime}}\left(p^{\prime 4} \tau^{\prime} \Gamma\right)
$$

where $p^{\prime}$ denotes the comoving particle momentum, with $p^{\prime} \simeq p^{\prime 0}$ for the energetic particles considered here, $\tau^{\prime} \simeq \lambda^{\prime} / c$ is the mean scattering time, and $\Gamma$ is the shear coefficient. In the strong scattering limit for quasi-isotropic diffusion in a turbulent environment (i.e. $\omega_{g}{ }^{\prime} \tau^{\prime} \lesssim 1$, with $\omega_{g}{ }^{\prime}$ the relativistic gyro-frequency measured in the comoving frame) we have (see Webb 1989, eq. 3.34)

$$
\Gamma=\frac{c^{2}}{30} \sigma_{\alpha \beta} \sigma^{\alpha \beta}
$$

where $\sigma_{\alpha \beta}$, with $\alpha, \beta=0,1,2,3$, is the (covariant) fluid shear tensor given by 1

$$
\sigma_{\alpha \beta}:=\nabla_{\alpha} u_{\beta}+\nabla_{\beta} u_{\alpha}+\dot{u}_{\alpha} u_{\beta}+\dot{u}_{\beta} u_{\alpha}-\frac{2}{3}\left(g_{\alpha \beta}+u_{\alpha} u_{\beta}\right) \nabla_{\delta} u^{\delta} .
$$

In this, $g_{\alpha \beta}$ denotes the (covariant) metric tensor and $\nabla_{\alpha}$ the covariant derivative. For spherical coordinates $x^{\alpha}=(c t, r, \theta, \phi)$, with $\phi$ the azimuthal and $\theta$ the polar angle, one has

$$
\left(g_{\alpha \beta}\right)=\operatorname{diag}\left(-1,1, \mathrm{r}^{2}, \mathrm{r}^{2} \sin ^{2} \theta\right) .
$$

The only non-vanishing connection coefficients (Christoffel symbols of the second kind) are then given by

$$
\begin{aligned}
& \Gamma_{22}^{1}=-r, \quad \Gamma_{21}^{2}=\Gamma_{12}^{2}=\Gamma_{13}^{3}=\Gamma_{31}^{3}=\frac{1}{r}, \quad \Gamma_{33}^{2}=-\sin \theta \cos \theta \\
& \Gamma_{33}^{1}=-r \sin ^{2} \theta, \quad \Gamma_{23}^{3}=\Gamma_{32}^{3}=\cot \theta
\end{aligned}
$$

Restricting ourselves to a time-independent, relativistic radial bulk flow velocity profile of the form

$$
u^{\alpha}=\gamma_{b}\left(1, v_{r}(r, \theta) / c, 0,0\right),
$$

where $\gamma_{b} \equiv \gamma_{b}(r, \theta)=\left[1-v_{r}(r, \theta)^{2} / c^{2}\right]^{-1 / 2}$ is the bulk Lorentz factor, the fluid four divergence becomes

$$
\nabla_{\beta} u^{\beta}=\frac{1}{r^{2}} \frac{\partial}{\partial r}\left(r^{2} \gamma_{b} v_{r} / c\right)
$$

while the only non-vanishing components of the fluid four acceleration $\dot{u}_{\alpha} \equiv u^{\beta} \nabla_{\beta} u_{\alpha}$ are

$$
\begin{aligned}
& \dot{u}_{0}=-\gamma_{b}^{4} \frac{v_{r}^{2}}{c^{3}} \frac{\partial v_{r}}{\partial r} \\
& \dot{u}_{1}=\gamma_{b}^{4} \frac{v_{r}}{c^{2}} \frac{\partial v_{r}}{\partial r} .
\end{aligned}
$$

\footnotetext{
${ }^{1}$ Note that this fixes a typographical sign error in eq.[A3] in (Rieger \& Duffy 2004).
} 
For the non-vanishing components of the shear tensor one then finds

$$
\begin{aligned}
\sigma_{00} & =\frac{4}{3} \gamma_{b}^{3} \frac{v_{r}^{2}}{c^{3}}\left(\gamma_{b}^{2} \frac{\partial v_{r}}{\partial r}-\frac{v_{r}}{r}\right), \\
\sigma_{01} & =\sigma_{10}=-\frac{4}{3} \gamma_{b}^{3} \frac{v_{r}}{c^{2}}\left(\gamma_{b}^{2} \frac{\partial v_{r}}{\partial r}-\frac{v_{r}}{r}\right), \\
\sigma_{11} & =\frac{4}{3 c} \gamma_{b}^{3}\left(\gamma_{b}^{2} \frac{\partial v_{r}}{\partial r}-\frac{v_{r}}{r}\right), \\
\sigma_{12} & =\sigma_{21}=\gamma_{b}^{3} \frac{1}{c} \frac{\partial v_{r}}{\partial \theta} \\
\sigma_{20} & =\sigma_{02}=-\gamma_{b}^{3} \frac{v_{r}}{c^{2}} \frac{\partial v_{r}}{\partial \theta} \\
\sigma_{22} & =\frac{2}{3} \gamma_{b} \frac{r^{2}}{c}\left(\frac{v_{r}}{r}-\gamma_{b}^{2} \frac{\partial v_{r}}{\partial r}\right), \\
\sigma_{33} & =\frac{2}{3} \gamma_{b} \frac{r^{2}}{c} \sin ^{2} \theta\left(\frac{v_{r}}{r}-\gamma_{b}^{2} \frac{\partial v_{r}}{\partial r}\right) .
\end{aligned}
$$

Noting that $\sigma^{\alpha \beta}=g^{\alpha \mu} g^{\delta \beta} \sigma_{\mu \delta}$, the relativistic shear coefficient becomes

$$
\Gamma=\frac{4}{45} \gamma_{b}^{2}\left[\left(\gamma_{b}^{2} \frac{\partial v_{r}}{\partial r}-\frac{v_{r}}{r}\right)^{2}+\frac{3}{4 r^{2}} \gamma_{b}^{2}\left(\frac{\partial v_{r}}{\partial \theta}\right)^{2}\right],
$$

which for non-relativistic flow speeds (i.e., $\gamma_{b} \rightarrow 1$ ) and $v_{r}$ independent of $r$ (and $\phi$ ), i.e. $v_{r} \equiv v_{r}(\theta)$, reduces to

$$
\Gamma=\frac{4}{45 r^{2}}\left[v_{r}^{2}+\frac{3}{4}\left(\frac{\partial v_{r}}{\partial \theta}\right)^{2}\right]
$$

It can be shown that this expression corresponds to the (non-relativistic) viscous transfer coefficient derived by Earl et al. (1988) (their eq. 7) when the latter is expressed in spherical coordinates and the corresponding velocity profile $\vec{v}=v_{r}(\theta) \vec{e}_{r}$ is applied.

For an energy-dependent scattering timescale of the form $\tau^{\prime} \propto p^{\prime \alpha}$, the shear flow acceleration coefficient, eq. (1), is given by

$$
\left\langle\frac{\Delta p^{\prime}}{\Delta t^{\prime}}\right\rangle_{s h}=(4+\alpha) \tau^{\prime} \Gamma p^{\prime}
$$

so that the characteristic acceleration timescale $t_{\text {acc }}(r, \theta) \simeq p^{\prime} /<\dot{p}^{\prime}>$ for gradual shear becomes

$$
t_{\mathrm{acc}}(r, \theta) \simeq \frac{45}{4(4+\alpha)} \frac{c}{\gamma_{b}^{2} \lambda^{\prime}}\left[\left(\gamma_{b}^{2} \frac{\partial v_{r}}{\partial r}-\frac{v_{r}}{r}\right)^{2}+\frac{3}{4 r^{2}} \gamma_{b}^{2}\left(\frac{\partial v_{r}}{\partial \theta}\right)^{2}\right]^{-1}
$$

where in the presence of a background magnetic field the particle mean free path formally has to be smaller than the gyro-radius to satisfy the strong scattering. Equation (21) exemplifies the characteristic inverse dependence, $t_{\mathrm{acc}} \propto 1 / \lambda^{\prime}$, on the particle mean free path. This is related to the fact that in a shear flow the average energy gain per scattering increases with increasing particle mean free path (Rieger \& Duffy 2006). 
Consider the simplified case where the radial flow velocity is only a function of polar angle $\theta$, so that in four-vector notation the flow speed is given by

$$
u^{\alpha}=\gamma_{b}\left(1, v_{r}(\theta) / c, 0,0\right)
$$

where $\gamma_{b} \equiv \gamma_{b}(\theta)=\left[1-v_{r}(\theta)^{2} / c^{2}\right]^{-1 / 2}$ is the bulk Lorentz factor of the flow. The associated (comoving) timescale for the shear flow acceleration of particles then becomes

$$
t_{\mathrm{acc}}(r, \theta) \simeq \frac{45}{4(4+\alpha)} \frac{c}{\lambda^{\prime}} \frac{r^{2}}{\gamma_{b}^{2}\left[v_{r}^{2}+0.75 \gamma_{b}^{2}\left(\partial v_{r} / \partial \theta\right)^{2}\right]},
$$

where $r$ is the radial coordinate measured in the cosmological rest frame, $\lambda^{\prime} \propto p^{\prime \alpha}$ is the particle mean free path, and $p^{\prime}$ is the particle momentum in the comoving (jet) frame. As the jet flow is diverging and streamlines are separating, the acceleration timescale increases with the square of the radial coordinate $r$.

\section{Flow velocity profiles and related energy losses}

By means of application, let us consider three different bulk flow velocity profiles $v_{r}(\theta)=$ $\sqrt{1-1 / \gamma_{b}(\theta)^{2}}$ parameterized in terms of $\gamma_{b}$ (cf. also Zhang \& Mészáros 2002, Kumar \& Granot 2003, Zhang et al. 2004, for instantiation in the case of GRBs), i.e., a power-law model, where $\gamma_{b}$ is powerlaw function of $\theta$ outside a core of opening angle $\theta_{c}$, i.e.,

$$
\gamma_{b}(\theta)=1+\left(\gamma_{b 0}-1\right)\left(1+\left[\frac{\theta}{\theta_{c}}\right]^{2}\right)^{-b / 2},
$$

with $1.5<b \lesssim 2$, a Gaussian profile with

$$
\gamma_{b}(\theta)=1+\left(\gamma_{b 0}-1\right) \exp \left(-\frac{\theta^{2}}{2 \theta_{c}^{2}}\right)
$$

and a Fermi-Dirac-type (top-hat) profile

$$
\gamma_{b}(\theta)=1+\left(\gamma_{b 0}-1\right)\left(1+\exp \left[-\beta_{c}\right]\right) /\left(1+\exp \left[\beta_{c}\left(\frac{\theta}{\theta_{c}}-1\right)\right]\right)
$$

with $\beta_{c}>0$, and where $\gamma_{b 0}$ denotes the Lorentz factor at the jet axis ( $\gamma_{b 0} \lesssim 50$ for AGN), and typically $\theta_{c} \lesssim 0.1 \mathrm{rad}$. Particle energization in these flow profiles then competes with conventional energy-loss processes. 


\subsection{Adiabatic Losses}

For the corresponding adiabatic energy changes one finds (using eq. 8)

$$
\left\langle\frac{\Delta p^{\prime}}{\Delta t^{\prime}}\right\rangle_{a d}:=-\frac{p^{\prime} c}{3} \nabla_{\beta} u^{\beta}=-\frac{p^{\prime}}{3}\left(2 \gamma_{b} \frac{v_{r}}{r}+\gamma_{b}^{3} \frac{\partial v_{r}}{\partial r}\right)=-\frac{2 p^{\prime}}{3} \gamma_{b} \frac{v_{r}}{r}
$$

where the last relation holds for $v_{r}$ independent of $r$. This gives the ratio of viscous gain versus adiabatic losses to

$$
\frac{\left\langle\frac{\Delta p^{\prime}}{\Delta t^{\prime}}\right\rangle_{s h}}{\left|\left\langle\frac{\Delta p^{\prime}}{\Delta t^{\prime}}\right\rangle_{a d}\right|}=\frac{2}{15}(4+\alpha) \gamma_{b}\left(\frac{\lambda^{\prime}}{r}\right)\left(\frac{v_{r}}{c}+\frac{3}{4} \gamma_{b}^{2} \frac{c}{v_{r}}\left(\frac{1}{c} \frac{\partial v_{r}}{\partial \theta}\right)^{2}\right)
$$

Hence, one expects viscous shear energization in the diffusion regime $\left(\lambda^{\prime}<r\right)$ for the present application (radially expanding flows, no azimuthal component) to be important only in the relativistic regime. Figures 1 and 2 show examples of the flow profile and energization ratio assuming $\gamma_{0}=30$. For this case, at a given $r$, only particles with $\lambda^{\prime} \gtrsim 0.03 r$ (power-law profile), $\lambda^{\prime} \gtrsim 0.002 r$ (Gaussian profile), and $\lambda^{\prime} \gtrsim 10^{-3} r$ (Fermi-Dirac) can get efficiently accelerated. Efficient acceleration thus needs energetic seed particles and is usually difficult to achieve for electrons unless the magnetic field is weak.

Depending on the shape of the velocity profile, particles are more easily accelerated (i.e., require less injection energy) at different angular scales, i.e., not necessarily in the innermost $\left(\theta \lesssim \theta_{c}\right)$ region. This becomes particularly apparent for the chosen Gaussian and the Fermi-Dirac type profile, where the shear gain to adiabatic loss ratio (Fig. 2) peaks at $\theta \simeq(2-3) \theta_{c}$. This could in principle introduce more complex emission features (see below) and support, for example, some ridge-line structure or a limb-versus centrally-brightened morphology (see e.g. Nagai et al. 2014, Boccardi et al. 2016, for recent exemplary findings in the context of 3C 84 and Cygnus A, respectively) in cases where potential differences in Doppler boosting are effectively compensated by more efficient particle acceleration.

For illustration, Fig. 4 shows two possible (optically-thin) intensity maps ( $I=\int \epsilon d l_{l o s}$, with $d l_{l o s}$ the line of sight element) for a jet possessing a Fermi-Dirac bulk flow profile and inclined at viewing angle $i=10^{\circ}$ in the case where particle injection across the jet is kept constant or varied with polar angle approximately following Fig. 2, For simplicity, it is assumed that the number density of non-thermal electrons (with differential energy distribution described by a power law of index $\alpha=2$ ) is proportional to the plasma density $n_{e} \propto \rho \propto 1 / z^{2}$ (where $z$ is the distance along the jet axis) and that the comoving emissivity is synchrotron type, $\epsilon^{\prime} \propto \rho^{\prime} B^{\prime(\alpha+1) / 2}$, with $\rho^{\prime}=\rho / \gamma_{b}$, $B^{\prime}=B / \gamma_{b}$ (perpendicular scaling, with $\left.B(z) \propto 1 / z\right)$ and $\epsilon=D^{(\alpha+3) / 2} \epsilon^{\prime}$ and $D$ the Doppler factor. In such a case, efficient shear acceleration leads to the appearance of a more prominent off-axis (ridge-line) structure.

If the above-mentioned conditions (Eq. 28) are satisfied, acceleration always proceeds (up to a factor of the order of unity) on timescales shorter (faster) than the relevant dynamical timescale 


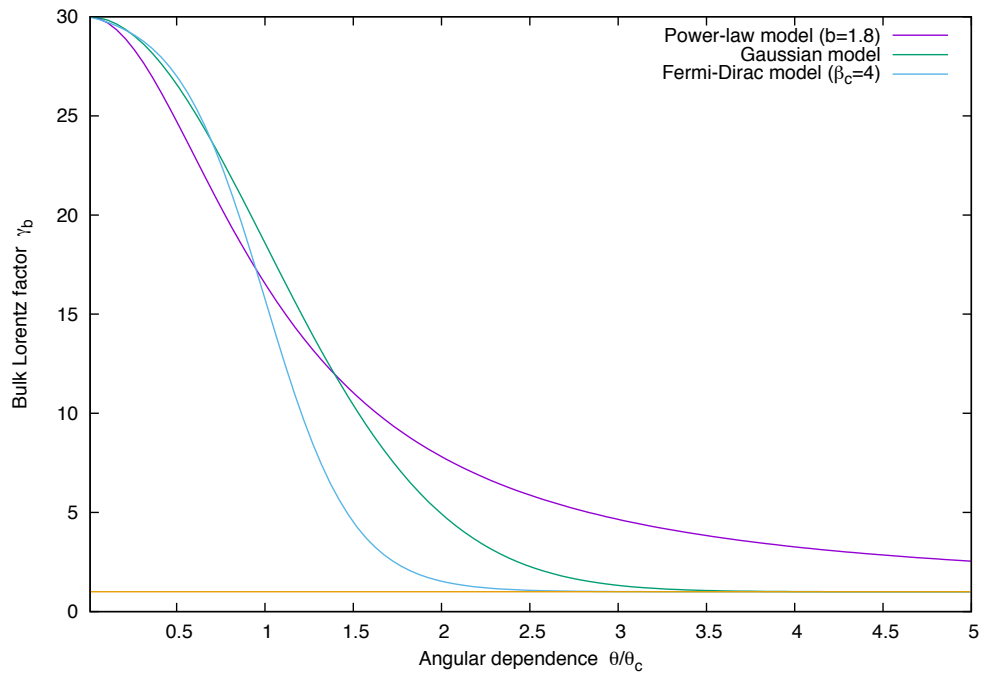

Fig. 1.- Illustration of the evolution of the bulk Lorentz factor $\gamma_{b}$ with polar angle $\theta$ for a power-law $(b=1.8)$, Gaussian and Fermi-Dirac type $\left(\beta_{c}=4\right)$ profile, respectively, assuming $\gamma_{b 0}=30$.

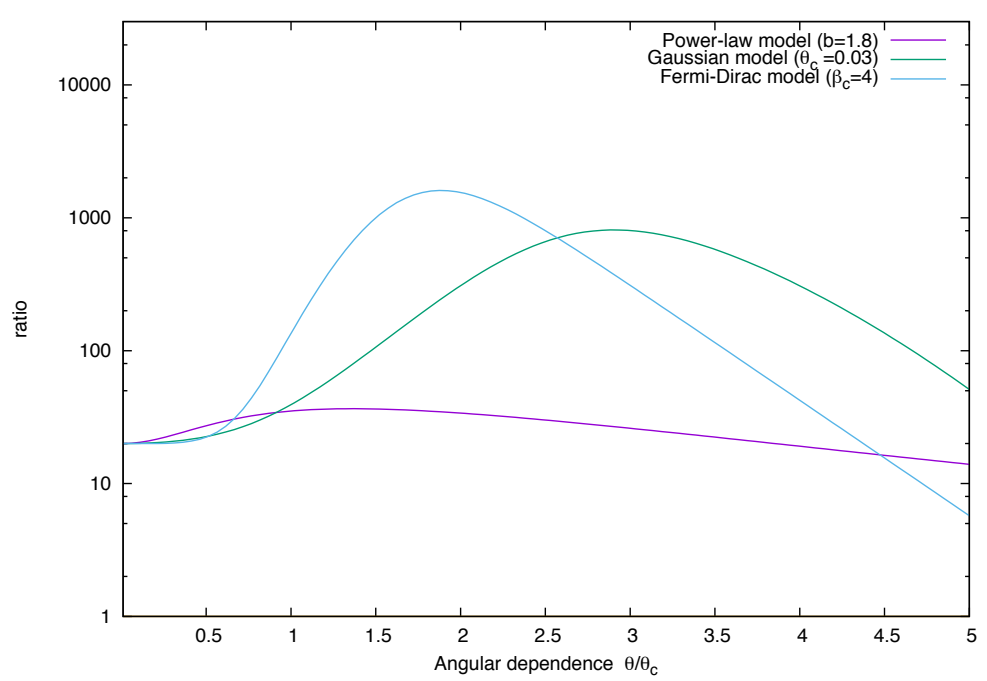

Fig. 2.- Associated ratio $\frac{\left.<\dot{p}^{\prime}\right\rangle_{s h}}{\left|\left\langle\dot{p}^{\prime}\right\rangle_{a d}\right|} \times\left(\frac{r}{\lambda^{\prime}}\right)$ between energization by viscous shear and adiabatic losses for the profiles given in Fig. 1, illustrated for $\theta_{c}=0.03$ [rad]. 
$t_{d}^{\prime} \sim \frac{r}{c \gamma_{b}}$. Hence, as a particle moves out along $r$, it will (in the absence of significant radiative losses) continue to get further accelerated to higher energies until it eventually leaves the flow by cross-field escape.

\subsection{Synchrotron Losses}

In the presence of magnetic fields, energetic charged particles will undergo synchrotron losses given by

$$
\left\langle\frac{\Delta p^{\prime}}{\Delta t^{\prime}}\right\rangle_{\mathrm{syn}}=-\frac{4}{9} \frac{q^{4}}{m^{2} c^{4}} \gamma^{\prime 2} B^{\prime 2}
$$

with $m$ the particle's rest mass, $q=e$ its charge, and $\gamma^{\prime}$ its Lorentz factor. We assume the background magnetic field to scale with radius $B(r)^{\prime}=B_{0}^{\prime}\left(r_{0} / r\right)^{a}$ with $0<a \leq 2$ (typically $a \sim 1)$. The ratio of shear gain to synchrotron losses then becomes

$$
\frac{\left\langle\frac{\Delta p^{\prime}}{\Delta t^{\prime}}\right\rangle_{s h}}{\left|\left\langle\frac{\Delta p^{\prime}}{\Delta t^{\prime}}\right\rangle_{\mathrm{syn}}\right|}=\frac{(4+\alpha)}{5}\left(\frac{\lambda^{\prime}}{r_{\text {gyro }}^{\prime}}\right) \frac{m^{4} c^{8}}{e^{5} B_{0}^{\prime 3}} \frac{1}{r_{0}^{2}} \gamma_{b}^{2}\left(\frac{r}{r_{0}}\right)^{3 a-2}\left(\frac{v_{r}^{2}}{c^{2}}+\frac{3}{4} \gamma_{b}^{2}\left(\frac{1}{c} \frac{\partial v_{r}}{\partial \phi}\right)^{2}\right)
$$

where $r_{g y r o}^{\prime}=\gamma^{\prime} m c^{2} /\left(e B^{\prime}\right)$ is the comoving gyro-radius of the particle. Using characteristic (conical jet-type) scaling numbers in the AGN context (and $\alpha \simeq 1$ ), this gives

$$
\begin{aligned}
\frac{\left\langle\frac{\Delta p^{\prime}}{\Delta t^{\prime}}\right\rangle_{s h}}{\left|\left\langle\frac{\Delta p^{\prime}}{\Delta t^{\prime}}\right\rangle_{\text {syn }}\right|} & \simeq 2 \times 10^{-10}\left(\frac{\lambda^{\prime}}{r_{\text {gyro }}^{\prime}}\right)\left(\frac{m}{m_{e}}\right)^{4}\left(\frac{10^{3} \mathrm{G}}{B_{0}^{\prime}}\right)^{3}\left(\frac{10^{13} \mathrm{~cm}}{r_{0}}\right)^{2}\left(\frac{\gamma_{b}}{30}\right)^{2}\left(\frac{r}{r_{0}}\right)^{3 a-2} \\
& \times\left(\frac{v_{r}^{2}}{c^{2}}+\frac{3}{4} \gamma_{b}^{2}\left(\frac{1}{c} \frac{\partial v_{r}}{\partial \theta}\right)^{2}\right) .
\end{aligned}
$$

Hence, if $\lambda^{\prime}$ scales with the gyro-radius, energetic protons $\left(m=m_{p}\right)$ are expected to experience efficient acceleration right from the start, almost independently of the magnetic field scaling. On the other hand, for the chosen magnetic field dependence (only on $r$ ), electrons $\left(m=m_{e}\right)$ will only be efficiently accelerated if the magnetic field becomes sufficiently weak, e.g., for $a=1.5$ on scales $r \gtrsim 5 \times 10^{3} r_{0}$. This is illustrated in Fig. 3 where the synchrotron ratio factor $d_{s}:=$ $\gamma_{b}^{2}\left(v_{r}^{2} / c^{2}+[3 / 4]\left[1 / c^{2}\right] \gamma_{b}^{2}\left[\partial v_{r} / \partial \theta\right]^{2}\right)$ is plotted for the considered flow profiles. In reality, however, one may expect the magnetic field to also reveal some $\theta$-dependence, probably decreasing with $\theta$, thereby facilitating the acceleration of particles further away from the axis. 


\subsection{Inverse Compton Losses}

For electrons, inverse Compton scattering (Thomson regime) could lead to additional cooling effects,

$$
\left\langle\frac{\Delta p^{\prime}}{\Delta t^{\prime}}\right\rangle_{\mathrm{IC}}=-\frac{4}{3} \sigma_{T} \gamma^{\prime 2} U_{p h}^{\prime}
$$

where $\sigma_{T}$ is the Thomson cross-section and $U_{p h}^{\prime}$ the energy density of the target photons as seen in the comoving frame. A non-reducible photon field is provided by the $\operatorname{CMB}\left(U_{C M B}=a T_{C M B}^{4} \simeq\right.$ $\left.4.2 \times 10^{-13} \mathrm{erg} \mathrm{cm}^{-3}[1+z]^{4}\right)$. With $U_{p h}^{\prime}=\gamma_{b}^{2} U_{p h}$, the ratio of shear energization to Compton losses becomes

$$
\begin{aligned}
\frac{\left\langle\frac{\Delta p^{\prime}}{\Delta t^{\prime}}\right\rangle_{s h}}{\left|\left\langle\frac{\Delta p^{\prime}}{\Delta t^{\prime}}\right\rangle_{\mathrm{IC}}\right|} & \simeq 10^{4}\left(\frac{\lambda^{\prime}}{r_{\text {gyro }}^{\prime}}\right)\left(\frac{r_{0}}{r}\right)^{2-a}\left(\frac{10^{13} \mathrm{~cm}}{r_{0}}\right)^{2}\left(\frac{10^{3} \mathrm{G}}{B_{0}^{\prime}}\right)\left(\frac{U_{C M B}}{U_{p h}}\right) \\
& \times\left(\frac{v_{r}^{2}}{c^{2}}+\frac{3}{4} \gamma_{b}^{2}\left(\frac{1}{c} \frac{\partial v_{r}}{\partial \theta}\right)^{2}\right),
\end{aligned}
$$

using the same scaling for the magnetic field as above. The Compton ratio factor $d_{C}:=\left(v_{r}^{2} / c^{2}+\right.$ $\left.[3 / 4]\left[1 / c^{2}\right] \gamma_{b}^{2}\left[\partial v_{r} / \partial \theta\right]^{2}\right)=d_{s} / \gamma_{b}^{2}$ is illustrated in Fig. 3. Accordingly, once the magnetic field becomes sufficiently weak so that electron acceleration can overcome synchrotron losses $\left(<\dot{p}^{\prime}\right\rangle_{s h}$ $/\left|<\dot{p}^{\prime}>_{\text {syn }}\right| \propto r^{3 a-2}$, shear energization is expected to continue out to larger distances until Compton losses set in $\left(<\dot{p}^{\prime}>_{s h} /\left|<\dot{p}^{\prime}>_{\mathrm{IC}}\right| \propto 1 / r^{2-a}\right)$.

\section{Discussion and Conclusion}

Shear flows are ubiquitous in astrophysical environments and potential sites of non-thermal particle acceleration. Relativistic outflows are in fact known to be conducive to efficient Fermi type shear particle acceleration (e.g., Ostrowski 2000, Rieger \& Duffy 2004). In the present paper, we have explored the potential of expanding relativistic outflows to boost energetic particles to higher energies. To this end, a set of simplified (azimuthally symmetric) conically expanding flow profiles (power-law, Gaussian and Fermi-Dirac-type) has been examined where the outflow bulk Lorentz factor is solely a function of polar angle. When applied to the AGN context, the results show that for the acceleration mechanism to overcome radiative and non-radiative losses and to work efficiently, the injection of pre-accelerated seed particles is required. This could in general be achieved by first-order shock or stochastic second-order Fermi processes. In this sense, shear acceleration would resemble a two-stage process for further particle energisation beyond the common limit. Depending on the shape of the flow profile, particles are more easily accelerated (i.e., require less injection energy) at different angular scales, i.e., not necessarily in the innermost core region close to the axis. This could in principle introduce different jet emission features (e.g., core versus

off-axis ridge-line structures or limb-brightening) and allow for a variety in jet appearance. Once operative, gradual shear acceleration proceeds on a timescale inversely proportional to the particle 
mean free path, $t_{\mathrm{acc}} \propto 1 / \lambda^{\prime}$. For a gyro-dependent particle mean free path, $\lambda^{\prime} \propto \gamma^{\prime}$, this gives the same scaling as synchrotron losses, $t_{\mathrm{acc}} / t_{\mathrm{syn}}=$ const. so that once started, synchrotron losses will not be able to further constrain particle acceleration, while for electrons Compton losses in an expanding flow might perhaps do so on larger scale.

From a methodological point of view, numerical studies focusing on the diffusive transport and acceleration of particles in turbulent fields (e.g. O'Sullivan et al. 2009) and the excitation of a largescale shear dynamo (e.g. Yousef et al. 2008) become of particular interest to adequately quantify the potential of shear particle acceleration on the relevant scales.

We note that shear acceleration in AGN jets seems in principle capable of accounting for continued acceleration and related extended emission. The inverse dependence on the particle mean free path makes shear acceleration a preferred mechanism for the acceleration of hadrons and provides some further weight to the relevance of AGN jets for our understanding of the origin of the recently detected high-energy (PeV) neutrinos (e.g. Becker Tjus et al. 2014; Tavecchio \& Ghisellini 2015) and the production of extreme cosmic-rays (e.g. Lemoine 2013).

F.M.R. kindly acknowledges support by a DFG Heisenberg Fellowship (RI 1187/4-1).

\section{REFERENCES}

Aloy, M.A., and Mimica, P. 2008, ApJ, 681, 84

Becker Tjus, J., Eichmann, B., Halzen, F. et al. 2014, PhRvD, 89, 123005

Bell, A.R. 2013, APh, 43, 56

Boccardi, B. et al. 2016, A\&A, 585, 33

Earl, J.A., Jokipii, J.R., \& Morfill, G. 1988, ApJ, 331, L91

Georganopoulos, M. and Kazanas 2003, D., ApJ, 594, L27

Ghisellini, G., Tavecchio, F. and Chiaberge, M. 2005, A\&A, 432, 401

Grismayer, T., Alves, E.P., Fonseca, R.A. and Silva, L.O. 2013, PPFC, 55, 124031

Hawley, J.F., Fendt, C., Hardcastle, M. et al. 2015, SSRv, 191, 441

Jokipii, J.R., \& Morfill, G.E. 1990, ApJ, 356, 255

Kumar, P., \& Granot, J. 2003, ApJ, 591, 1075

Laing, R.A. and Bridle, A.H. 2013, MNRAS, 432, 1114

Lemoine, M. 2013, JPhCs, 409, 2007 
Levinson, A. 2007, ApJ, 671, L29

Liang, E., Boettcher, M. and Smith, I. 2013, ApJ, 766, L19

Lister, M.L. et al. 2009, AJ, 138, 1874

McKinney, J.C. 2006, MNRAS, 368, 1561

Nagai, H. et al. 2014, ApJ, 785, 53

Ohira, Y. 2013, ApJ, 767, 16

Ostrowski, M. 2000, MNRAS, 312, 579

Piner, B.G. et al. 2012, ApJ, 758, 84

Piner, B.G., and Edwards, P.G. 2013, EPJWC 6104021

Porth, O. and Fendt, C. 2010, ApJ, 709, 1100

Rieger, F.M., \& Duffy, P. 2004, ApJ, 617, 155

Rieger, F.M., \& Duffy, P. 2005, ApJ, 632, L21

Rieger, F.M., \& Duffy, P. 2006, ApJ, 652, 1044

Rieger, F.M. and Aharonian, F.A. 2012, MPLA, 27, 12300301

Sahayanathan, S. 2009, MNRAS, 398, 49

O’Sullivan, S., Reville, B., \& Taylor, A. M. 2009, MNRAS, 400, 248

Tavecchio, F., \& Ghisellini, G. 2015, MNRAS, 451, 1502

Webb, G.M. 1989, ApJ, 340, 1112

Yousef, T. A., Heinemann, T., Rincon, F., et al. 2008, Astronomische Nachrichten, 329, 737

Zhang, B., \& Mészáros, P. 2002, ApJ, 571, 876

Zhang, B., Dai, X., Lloyd-Ronning, N.M., \& Mészáros, P. 2004, ApJ, 601, L119 

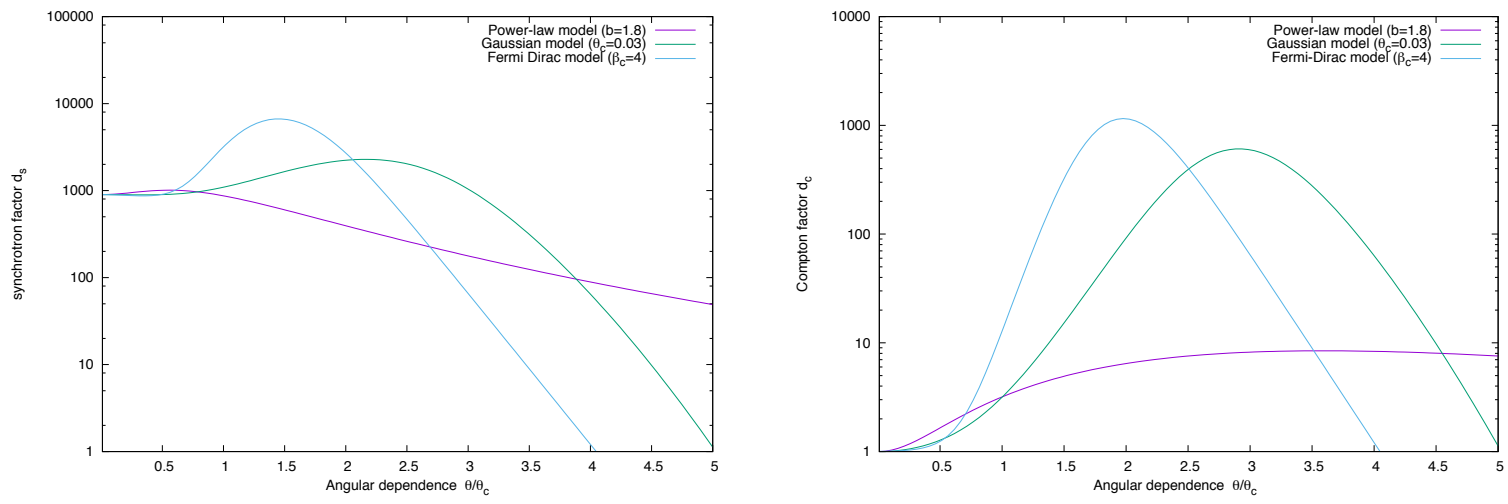

Fig. 3.- Left: Associated synchrotron ratio factor $d_{s}$ for shear energization versus synchrotron cooling for the flow profiles given in Fig. 1, illustrated for $\theta_{c}=0.03$ [rad]. Right: Associated Compton ratio factor $d_{C}$ for shear energization versus Compton cooling.
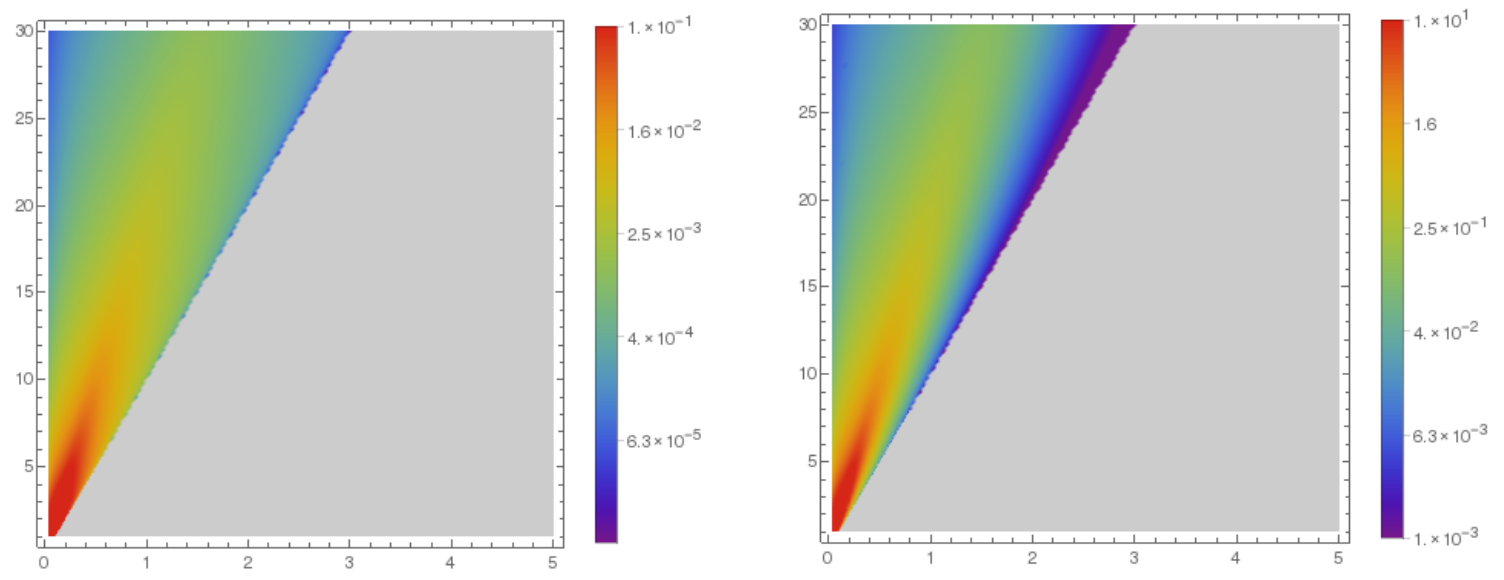

Fig. 4. - Illustration of intensity maps showing the possible change in appearance for a relativistically expanding jet with a Fermi-Dirac type profile (using $\gamma_{b 0}=30, \beta_{c}=4$ and $i=10^{\circ}$ for the inclination of the main $z$-axis to the line of sight) where the rate of particle injection is kept constant (left) and varied (right) as a function (quasi-Gaussian centred around the peak value in Fig. 2) of the polar angle $\theta$, respectively. 